\title{
Conversion of cellulose $I \alpha$ to $I \beta$ via a high temperature intermediate (I-HT) and other cellulose phase transformations
}

\author{
James F. Matthews • Michael E. Himmel • \\ Michael F. Crowley
}

Received: 1 June 2011 / Accepted: 10 October 2011/Published online: 6 November 2011

(C) Springer Science+Business Media B.V. (outside the USA) 2011

\begin{abstract}
The observation that the hydrothermal conversion of cellulose $\mathrm{I} \alpha$ to cellulose $\mathrm{I} \beta$ is irreversible has been assumed to be due to the relative free energy of these polymorph phases. We propose an alternative explanation: when cooling the high temperature phase, the barrier to forming $\mathrm{I} \beta$ is much smaller than the barrier to forming $\mathrm{I} \alpha$, so kinetics favor the formation of $\mathrm{I} \beta$. This explanation is consistent with all available experimental data, and is consistent with the general observation of polymer solid-solid phase transformations via metastable intermediate states. While cellulose $\mathrm{I} \beta$ may be lower in free energy than $\mathrm{I} \alpha$, this has not been shown experimentally. Phase transformations of other cellulose polymorphs may be subject to similar kinetic effects when converted via metastable intermediate states.
\end{abstract}

Keywords Phase transformation - Polymorph . Cellulose I $\alpha$ I $\beta$ II III I IV IV IV I-HT alpha beta

\section{Introduction and background}

Heating cellulose $\mathrm{I} \alpha$ or $\mathrm{I} \beta$ produces a high temperature phase (Wada et al. 2003; Wada et al. 2010), which

J. F. Matthews $(\square) \cdot$ M. E. Himmel · M. F. Crowley $(\square)$

Biosciences Center, National Renewable Energy

Laboratory, Golden, CO, USA

e-mail: james.matthews@nrel.gov

M. F. Crowley

e-mail: michael.crowley@nrel.gov upon cooling produces cellulose $\mathrm{I} \beta$ (Yamamoto et al. 1989; Sugiyama et al. 1990; Debzi et al. 1991). This observation has widely been assumed to be evidence that the $\mathrm{I} \beta$ phase is lower in free energy than the $\mathrm{I} \alpha$ phase. However, although the assumed difference in free energy might be correct, this observation is not a measurement of relative thermodynamic stability. Comparing the cellulose $\mathrm{I} \alpha$ and $\mathrm{I} \beta$ crystal structures to the high temperature phase structure we call I-HT, we describe a pathway for the conversion of $\mathrm{I} \alpha$ to $\mathrm{I} \beta$ via I-HT. This proposed explanation does not depend on the relative free energy of the $\mathrm{I} \alpha$ and I $\beta$ polymorphs, which is yet to be determined experimentally.

At the molecular level cellulose $\mathrm{I} \alpha$ and $\mathrm{I} \beta$ share similar conformation and chain packing in the $\mathbf{a}-\mathbf{b}$ planes, but differ in layer packing along the $\mathbf{c}$ direction such that $\mathrm{I} \alpha$ has $\mathrm{P} 1$ symmetry and $\mathrm{I} \beta$ has $\mathrm{P} 2{ }_{1}$ symmetry (Nishiyama et al. 2002; Nishiyama et al. 2003). Kinetic trapping of $\mathrm{P} 21_{1}$ symmetry is a possible explanation for why cellulose $\mathrm{I} \beta$ is produced upon cooling the intermediate high temperature phase (Cheng and Keller 1998). Consistent with this hypothesis are the structural features of the high temperature phase we call I-HT (Matthews et al. 2011; Zhang et al. 2011). Figure 1a depicts the observed transformations between I $\beta$ and I-HT, and between I $\alpha$ and I-HT. In Fig. 1b, we propose a free energy landscape for these transformations at room temperature and under high temperature conditions. This proposed landscape accounts for the observed phenomenon of an irreversible I $\alpha$ to I $\beta$ transformation through kinetic effects. 
A

$\mathrm{I} \beta \rightleftharpoons \mathrm{I}-\mathrm{HT} \longleftarrow \mathrm{I} \alpha$

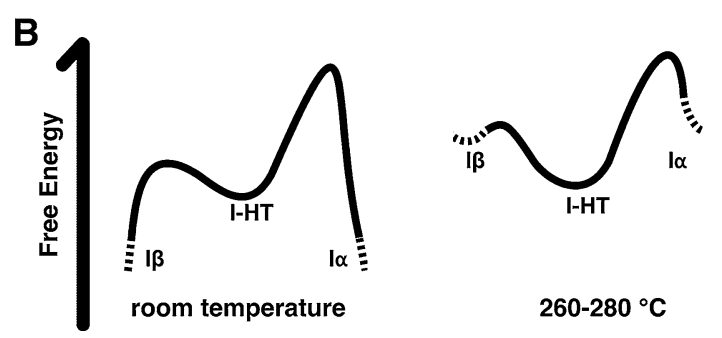

Fig. 1 a A diagram of the hydrothermal conversion pathways of cellulose I is shown. Cellulose $\mathrm{I} \alpha$ is transformed to $\mathrm{I} \beta$ through a high temperature phase intermediate, I-HT, which is more similar in structure to $\mathrm{I} \beta$ than to $\mathrm{I} \alpha$. b Proposed free energy landscapes for the transformation of cellulose $\mathrm{I} \alpha$ to $\mathrm{I} \beta$ at room temperature and at temperatures near $260-280{ }^{\circ} \mathrm{C}$ are shown. The relative free energy of the cellulose $\mathrm{I} \alpha$ and $\mathrm{I} \beta$ end point structures is unknown. At high temperature the intermediate I-HT structure is lower in free energy than both $\mathrm{I} \alpha$ and $\mathrm{I} \beta$, and the barriers to form the I-HT phase are relatively low. The barriers between I-HT and $\mathrm{I} \alpha$ are significantly higher than the barriers between I-HT and I $\beta$ due to the large-scale molecular motions required to move away from $\mathrm{I} \alpha$-like layer packing. Therefore, upon cooling we hypothesize the rate to form $\mathrm{I} \beta$ is much faster than the rate to form $\mathrm{I} \alpha$ due to the structural similarity of the $\mathrm{I} \beta$ and I-HT phases

This proposal does not hinge upon the relative thermodynamic stability of cellulose $\mathrm{I} \alpha$ and $\mathrm{I} \beta$. However, polymorph stability remains of practical interest for chemical modification or hydrolysis of cellulose, where $\mathrm{I} \alpha$ is found to be more susceptible than I $\beta$ (Hayashi et al. 1997a, b; Boisset et al. 1999; Sassi et al. 2000). It is unclear if this difference in susceptibility is due to differences in crystal surface morphology, a different surface versus interior distribution of these phases, or if it reflects a real difference in the reactivity of the polymorphs (Wada and Okano 2001; Horikawa and Sugiyama 2009; Beckham et al. 2011).

The conversion of cellulose $\mathrm{I} \alpha$ to $\mathrm{I} \beta$ is an activated process, requiring high temperatures and slow largescale molecular motions. To effectively transform cellulose $\mathrm{I} \alpha$ to $\mathrm{I} \beta$, large $\sim 20 \mathrm{~nm}$ diameter $\mathrm{I} \alpha$ rich microfibrils must be held at $260{ }^{\circ} \mathrm{C}$ for $30 \mathrm{~min}$ in $0.1 \mathrm{~N} \mathrm{NaOH}$, or at $280{ }^{\circ} \mathrm{C}$ for $60 \mathrm{~min}$ in nonpolar media or in inert atmosphere (Horii et al. 1987; Yamamoto et al. 1989; Debzi et al. 1991). In this temperature region, a high temperature structure of cellulose I is formed (Wada et al. 2003; Wada et al. 2010; Matthews et al. 2011; Zhang et al. 2011). This suggests that the high temperature phase is an intermediate state in the conversion of $\mathrm{I} \alpha$ to $\mathrm{I} \beta$ (Wada et al. 2003). The timescale for converting from $I \alpha$ to the high temperature phase suggests a significant barrier for this transformation. This transformation proceeds from the surface to the interior of large microfibrils over 30-60 min (Horikawa and Sugiyama 2009).

We note that it has not been shown definitively by experiment that $\mathrm{I} \alpha$ and $\mathrm{I} \beta$ heated to high temperature are structurally equivalent. This has likely not yet been demonstrated due to short holding time at the required temperature. Results from 2D-MWIR spectroscopy (Watanabe et al. 2007) indicate that the spectra might converge if, during the experiment in inert atmosphere, I $\alpha$ was held at $280{ }^{\circ} \mathrm{C}$ for $60 \mathrm{~min}$ instead of at $260{ }^{\circ} \mathrm{C}$ for $5 \mathrm{~min}$ (Debzi et al. 1991). Changes in spacing between layers during heating and subsequent cooling, as measured by X-ray diffraction, also indicate that convergence might occur if $I \alpha$ were held at sufficient temperature longer than 15 min (Wada et al. 2003). The alternative possibility, that heated $\mathrm{I} \alpha$ and $\mathrm{I} \beta$ maintain different structures when held for long enough time at sufficient temperatures to transform $\mathrm{I} \alpha$ to $\mathrm{I} \beta$ upon cooling, seems unlikely. The large-scale motions required to convert $\mathrm{I} \alpha$-like to $\mathrm{I} \beta$-like layer packing are most likely to occur when kinetic energy is highest.

\section{Cellulose I structure and symmetry}

Cellulose $\mathrm{I} \alpha$ and $\mathrm{I} \beta$ differ mainly in the stacking arrangement of hydrogen bonded layers, with $\mathrm{I} \alpha$ layers always displaced along the molecular axis by $+\mathbf{c} / 4$, whereas hydrogen bonded layers in cellulose $\mathrm{I} \beta$ alternate between $+\mathbf{c} / 4$ and $-\mathbf{c} / 4$ displacement (Sugiyama et al. 1991; Imai and Sugiyama 1998; Nishiyama et al. 2002; Nishiyama et al. 2003). This alternating layer arrangement in $\mathrm{I} \beta$ makes half of the inter-layer interactions identical to those in $\mathrm{I} \alpha$. A combined $\mathrm{I} \beta$ and $\mathrm{I} \alpha$ microfibril model showing this difference in relative layer stacking is shown in Fig. 2. For a homopolymer, a monomer is defined to be the smallest repeating constitutional unit. In cellulose, the monomer unit is anhydroglucose. In Fig. 2, two anhydroglucose monomers in each layer are shown in black larger than the rest of the chain. These 


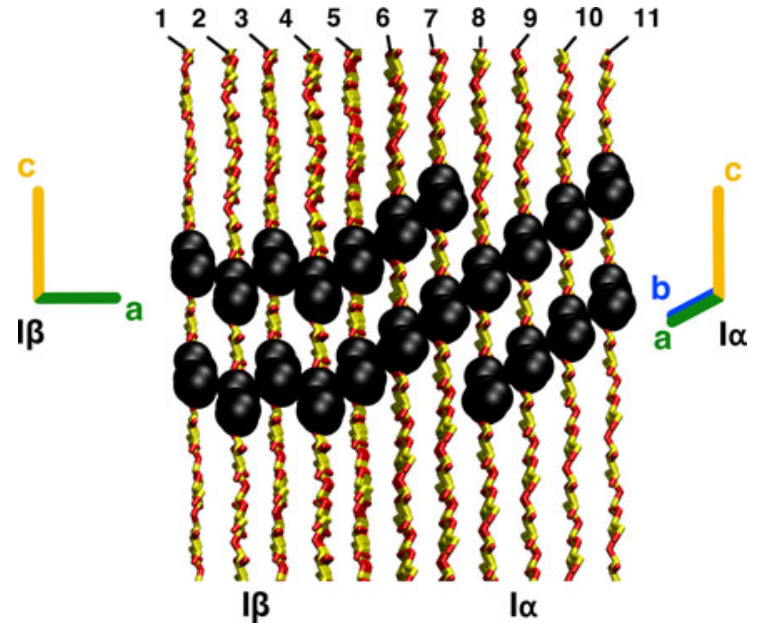

Fig. 2 A model microfibril containing both cellulose $\mathrm{I} \alpha$ and $\mathrm{I} \beta$ constructed to illustrate layer packing, as viewed across hydrogen bonded layers with the chain axis vertical. Layers in $\mathrm{I} \alpha$ are displaced along the molecular axis by $+\mathbf{c} / 4$, whereas layers in cellulose $\mathrm{I} \beta$ alternate between $+\mathbf{c} / 4$ and $-\mathbf{c} / 4$ displacement. Black spheres indicate monomers where the primary alcohol group is facing out of the page. For each monomer, a translation by $\pm \mathbf{c} / 4$ or a rotation by $180^{\circ}$ about the c-axis would effectively switch the direction each primary alcohol group faces. Fig. 3 shows in detail the effect of a crystal rotation by $180^{\circ}$ on the relative orientation of monomers within neighboring layers of cellulose $\mathrm{I} \beta$ and $\mathrm{I} \alpha$. Layers 4 and 5 cannot be unambiguously designated as belonging exclusively to either the $\mathrm{I} \alpha$ or $\mathrm{I} \beta$ phase. Unit cell axes are shown to scale. Hydrogen atoms are omitted, and oxygen atoms are colored red. Layer numbers at top are used to describe an example $\mathrm{I} \alpha$ to $\mathrm{I} \beta$ transformation pathway in the main text

indicated monomers have the primary alcohol O6 group facing out of the page, and the monomer between these indicated monomers has the primary alcohol group facing into the page. Two layers cannot be unambiguously classified as $\mathrm{I} \alpha$ or $\mathrm{I} \beta$ based on relative layer displacement alone. Additionally, hydrogen bonds within layers of both cellulose $\mathrm{I} \beta$ and $\mathrm{I} \alpha$ at room temperature can be assigned one of two competing networks, but it is not clear whether this hydrogen bond disorder is static or dynamic, or whether it is an artifact due to other kinds of structural disorder (Nishiyama et al. 2002; Nishiyama et al. 2003, Langan et al. 2005).

To convert cellulose $\mathrm{I} \alpha$ to $\mathrm{I} \beta$, either some layers slide relative to the others by $\mathbf{c} / 2$ or some chains rotate by $180^{\circ}$ (Hardy and Sarko 1996; Vietor et al. 2000; Nishiyama et al. 2003; Wada et al. 2003). For example, in the model microfibril containing both cellulose $\mathrm{I} \alpha$ and $\mathrm{I} \beta$ shown in Fig. 2 , if chains in layers $6,7,10$, and 11 were to slide by $\pm \mathbf{c} / 2$ or to rotate by $180^{\circ}$ around the chain axis (c-direction), the entire microfibril would have I $\beta$ layer packing. Many pathways are possible to accomplish this transformation. The experimentally determined layer spacing at elevated temperature is more consistent with the hypothesis of a layer slip $\mathrm{I} \alpha$ to $\mathrm{I} \beta$ conversion mechanism than with a mechanism of chain rotation (Wada et al. 2010). Chain rotation by $180^{\circ}$ would likely require a lattice spacing increase of the difference between the maximum and the minimum width of a cellulose chain, approximately $1.5 \AA(5-3.5 \AA)$. The observed increase in layer spacing at $280{ }^{\circ} \mathrm{C}$ is approximately $0.5 \AA$.

Another way to switch between $\mathrm{I} \alpha$-like and $\mathrm{I} \beta$-like layer packing in a single chain is an unusual conformation of the glycosidic linkage. The glycosidic linkages of cellulose in the $\mathrm{I} \alpha$ and $\mathrm{I} \beta$ structures have the syn conformation. A single anti conformation of a glycosidic linkage in a cellulose chain would produce a $180^{\circ}$ rotation of the chain. Alternation between $\mathrm{I} \alpha$ and $\mathrm{I} \beta$ packing along single microfibrils at $\sim 50 \mathrm{~nm}$ length scale has been observed, which may be partly explained by occasional chain breaks, or by these unusual conformations of the glycosidic linkage (Sugiyama et al. 1991; Debzi et al. 1991; Imai and Sugiyama 1998).

I-HT structures proposed from simulation (Matthews et al. 2011; Zhang et al. 2011), which are consistent with diffraction studies (Wada et al. 2010) and other experimental studies (Horikawa et al. 2009), show alternating $+\mathbf{c} / 4$ and $-\mathbf{c} / 4$ layer displacement and approximate $\mathrm{P} 2{ }_{1}$ screw symmetry similar to the cellulose $\mathrm{I} \beta$ crystal structure. In these structures there are two chains per unit cell, which are located at the origin of the unit cell and at the center of the unit cell. These special chain locations within the unit cell, combined with $\mathrm{P} 2{ }_{1}$ symmetry about the chain axis (cdirection), make each chain have single equivalent monomers repeating $-\mathrm{B}_{\text {origin }}-\mathrm{B}_{\text {origin }}{ }^{\prime}-$ and $-\mathrm{B}_{\text {center }}{ }^{-}$ $\mathrm{B}_{\text {center }}$ - along the length of the chains in $\mathrm{I} \beta$. In contrast, in $\mathrm{I} \alpha$ there is $\mathrm{P} 1$ symmetry (translational symmetry only) and one chain per unit cell with two non-equivalent monomers repeating $-\mathrm{A}_{1}-\mathrm{A}_{2}-\mathrm{A}_{1}{ }^{\prime}-$ $\mathrm{A}_{2}{ }^{\prime}-$ along the length of each chain (Nishiyama et al. 2002; Nishiyama et al. 2003; Kono and Numata 2006). There is some conformational disorder in the I-HT structures reported in Matthews et al. (2011), but in 

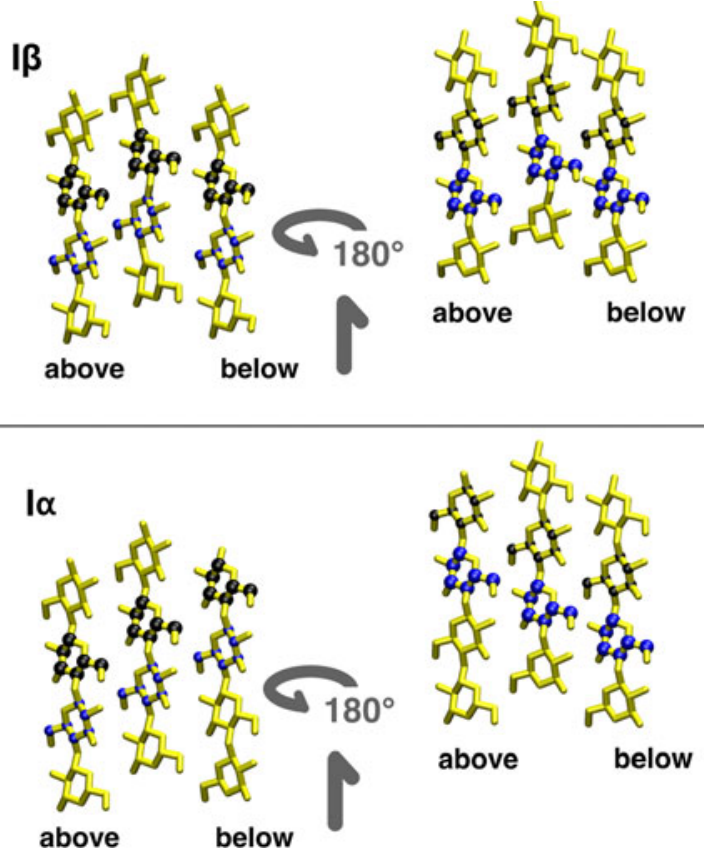

Fig. 3 Three chains are shown for $\mathrm{I} \beta$ and $\mathrm{I} \alpha$, belonging to $(110)_{\beta}$ or $(100)_{\alpha}$ planes. For $\mathrm{I} \beta$, two origin chains are shown above and below one center chain. The effect of a $180^{\circ}$ rotation about the chain axis (c-direction) and a translation of $\mathbf{c} / 2$ on the topology of neighboring interactions is different for $\mathrm{I} \beta$ and $\mathrm{I} \alpha$. By definition this transformation acting on $\mathrm{I} \beta$, which has $\mathrm{P} 2_{1}$ symmetry, preserves the topology of local interactions for a given orientation and location of a monomer. For $\mathrm{I} \alpha$, which has P1 symmetry, this transformation reverses the orientation of neighboring monomers related by translational symmetry. This difference in layer packing is significant at elevated temperature where chains can tilt cooperatively to form $\mathrm{O} 6_{\text {center }}-\mathrm{O} 2_{\text {origin }}$ interlayer hydrogen bonds when there is $\mathrm{P} 21$ symmetry, but not when there is $\mathrm{I} \alpha$-like P1 symmetry. Hydrogen atoms are omitted

similar simulations by Zhang et al. (2011) there is less conformational disorder at slightly higher temperatures.

For each monomer along a given chain in the I-HT phase, and in cellulose $\mathrm{I} \beta$, which have $\mathrm{P} 2{ }_{1}$ symmetry, approximate 2 -fold symmetry in the chains make all interlayer interactions topologically identical. In cellulose $\mathrm{I} \alpha$, which has $\mathrm{P} 1$ symmetry, for each monomer there is a distinction between the layer 'above' and the layer 'below' the current layer. Figure 3 shows this difference in symmetry, where a rotation by $180^{\circ}$ about the c-axis preserves the topology of local interactions for $\mathrm{I} \beta$, but reverses the location of similar neighbors in $\mathrm{I} \alpha$.

\section{Why cellulose $I \alpha$ is transformed to $I \beta$ by thermal treatment}

Here we propose an explanation of why thermal treatment transforms cellulose $\mathrm{I} \alpha$ to cellulose $\mathrm{I} \beta$, but not the reverse transformation, by comparing the experimentally determined $\mathrm{I} \alpha$ and $\mathrm{I} \beta$ crystal structures to high temperature structures from simulation. Consistent with previously proposed mechanisms of this transformation (Hardy and Sarko 1996; Vietor et al. 2000; Nishiyama et al. 2003; Wada et al. 2003), we suggest that chain slip produces the change from $\mathrm{I} \alpha$ like to $\mathrm{I} \beta$-like layer packing. We hypothesize the reason this transformation appears to be irreversible is that $\mathrm{I} \alpha$-like layer packing at high temperature is not stable, so layers slip to produce $\mathrm{I} \beta$-like packing. Cooling therefore produces $\mathrm{I} \beta$, because pathways from I-HT leading back to I $\alpha$-like packing are significantly less kinetically favored than the pathway to $\mathrm{I} \beta$. This detail differs from previous proposals where the irreversible conversion was assumed to reflect lower free energy of the I $\beta$ structure, and where the structure of the high temperature intermediate and kinetics were not taken into account.

In the high temperature phase I-HT structures from simulation, chains in each layer are tilted such that the cooperative formation of $\mathrm{O}_{\text {center }}-\mathrm{O} 2_{\text {origin }}$ hydrogen bond interactions is possible, when combined with changes in hydroxymethyl orientation. In cellulose $\mathrm{I} \alpha$, this inter-layer O6-O2 hydrogen bond interaction is possible for every second monomer in every chain (such as those indicated with blue spheres at bottom in Fig. 3), but not all of these interactions can form at the same time. Cellulose $I \alpha$-like layer packing is not likely to be stable at high temperature because only each second monomer can form these inter-layer interactions, and only half of these can form at the same time in a competitive rather than cooperative manner. In I $\alpha$, half of the monomers can act as the O6 hydrogen bond partner or as the $\mathrm{O} 2$ partner, but not as both at the same time. In contrast, $\mathrm{I} \beta$-like layer packing at high temperature allows all monomers to form $\mathrm{O} 6_{\text {center }}-\mathrm{O} 2_{\text {origin }}$ hydrogen bonds cooperatively and simultaneously.

Figures 4 and 5 show chains from four neighboring layers of cellulose I $\beta$ (Nishiyama et al. 2002), I-HT (Matthews et al. 2011), and I $\alpha$ (Nishiyama et al. 2003), corresponding to $(110)_{\beta, \mathrm{HT}}$ and $(100)_{\alpha}$ planes, highlighting similarities and differences in chain and layer packing. Monomers are colored to indicate 

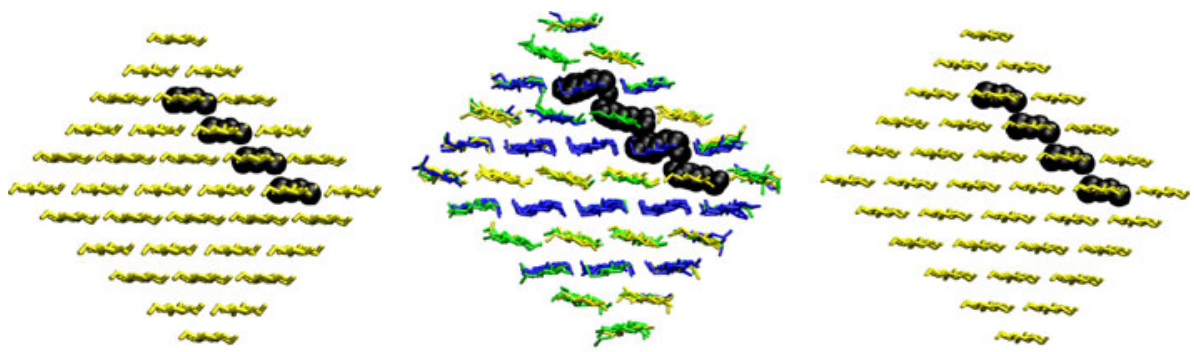

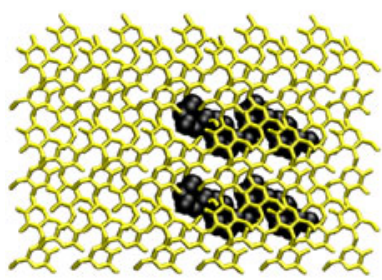

Iß

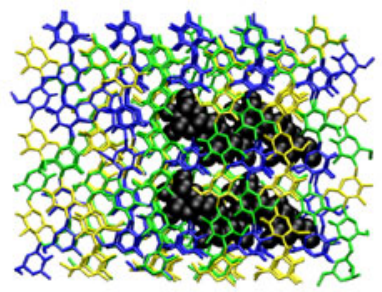

I-HT

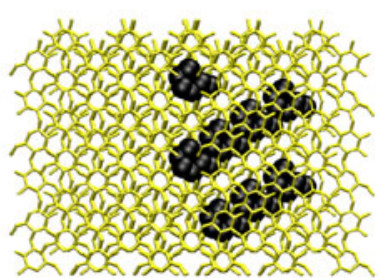

$1 \alpha$
Fig. 4 Two views of chains belonging to $(110)_{\beta, \mathrm{HT}}$ and $(100)_{\alpha}$ planes in 36 chain microfibril models. As in Fig. 2, two monomers in each indicated chain (with the primary alcohol group facing to the right) are illustrated with large black spheres. At top, viewed with chains into the page, cross-section chain packing in $\mathrm{I} \alpha$ and $\mathrm{I} \beta$ is nearly identical whereas chains tilt in the

hydroxymethyl orientation, with TG yellow, GT green, and GG blue (Matthews et al. 2006). For a given chain in $\mathrm{I} \beta$ and I-HT, interactions with monomers in adjacent layers are identical for each monomer along the chain due to $\mathrm{P} 2{ }_{1}$ symmetry. In contrast, for a given chain in $\mathrm{I} \alpha$, every second monomer along the chain has the same interactions with monomers in adjacent layers due to P1 symmetry. By comparing structural features of the high temperature phase and of cellulose $\mathrm{I} \alpha$ and $\mathrm{I} \beta$, the mechanism of the irreversible phase transition from $\mathrm{I} \alpha$ to $\mathrm{I} \beta$ through hydrothermal treatment can be proposed, as detailed in the following paragraphs.

The same number of $\mathrm{O} 6$ groups exist near to $\mathrm{O} 2$ groups in neighboring $(110)_{\beta, \mathrm{HT}}$ or $(100)_{\alpha}$ layers, but these possible hydrogen bond pairs are distributed differently. These O6-O2 pairs are distributed as each second monomer in every chain of $\mathrm{I} \alpha$, whereas in $\mathrm{I} \beta$ the O6 groups are every monomer in center layers and the $\mathrm{O} 2$ groups are every monomer in origin layers, as indicated by ovals in Fig. 5. At temperatures near 220-230 ${ }^{\circ} \mathrm{C}$, layer spacing begins to increase. Results from simulations show this increase in layer spacing is due to hydroxymethyl rotation coupled with chain tilting. For $\mathrm{I} \alpha$ at this temperature, we propose that chain tilt is not stable because it is impossible for all of the
I-HT structure. At bottom, viewed with chain axis vertical, layer packing in $\mathrm{I} \beta$ and I-HT is very similar with two chains per unit cell. The P1 layer packing in cellulose $\mathrm{I} \alpha$, when viewed from this direction, shows a repeating pattern after four layers as opposed to repeating after two layers in $\mathrm{I} \beta$ and I-HT

potential O6-O2 interlayer interactions to form at the same time. In $\mathrm{I} \alpha$, the distribution of these pairs would tend to cause the O6 side of every second monomer to tilt towards the $\mathrm{O} 2$ side in the layer above, but the $\mathrm{O} 2$ side of these same monomers would tend to make the chain tilt in the opposite direction. This makes these inter-layer interactions for $\mathrm{I} \alpha$-like layer packing competitive rather than cooperative as in $\mathrm{I} \beta$-like layer packing, so at most half of these interactions can form at the same time for $\mathrm{I} \alpha$-like layer packing. For $\mathrm{I} \beta$ at high temperature, alternating by layer chain tilt with $\mathrm{P} 2_{1}$ symmetry does allow all of these potential interlayer $\mathrm{O} 6_{\text {center }}-\mathrm{O} 2_{\text {origin }}$ interactions to form simultaneously.

We hypothesize that at sufficiently high temperatures (near $260{ }^{\circ} \mathrm{C}$ in $0.1 \mathrm{~N} \mathrm{NaOH}$ or near $280{ }^{\circ} \mathrm{C}$ in inert an atmosphere), $\mathrm{I} \alpha$-like packing is unstable due to conflicting requirements of chain tilt when interlayer O6-O2 hydrogen bonds form as shown in Fig. 6. Each second monomer along every chain in $\mathrm{I} \alpha$ can potentially form interlayer O6-O2 hydrogen bonds, but only to one neighboring layer as the $\mathrm{O} 6$ partner or to the other neighboring layer as the $\mathrm{O} 2$ partner. At these temperatures there is sufficient kinetic energy for $I \alpha$ layers to slip along the c-axis. This forms alternating $\pm \mathbf{c} / 4$ layer staggered packing as in $\mathrm{I} \beta$, and allows for the stable alternating-by-layer chain tilt pattern to 


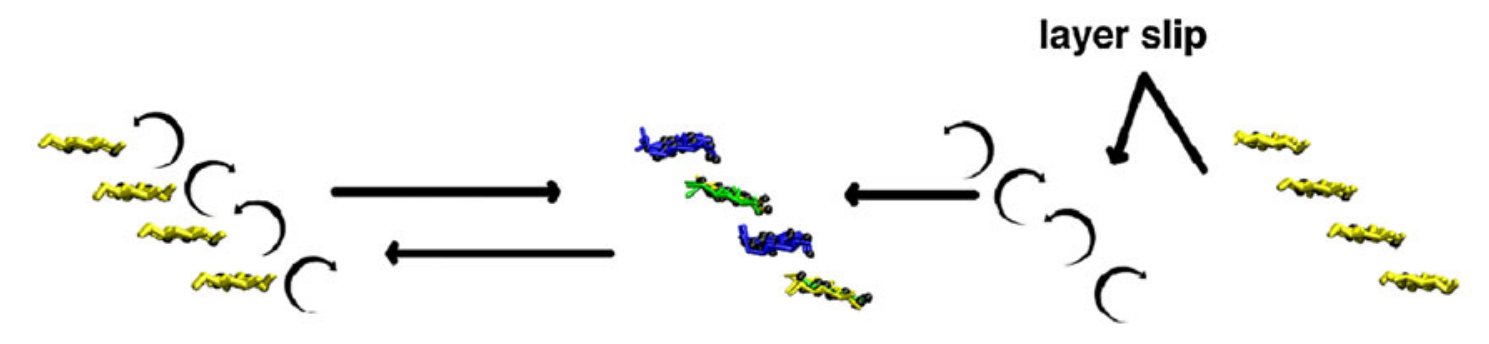

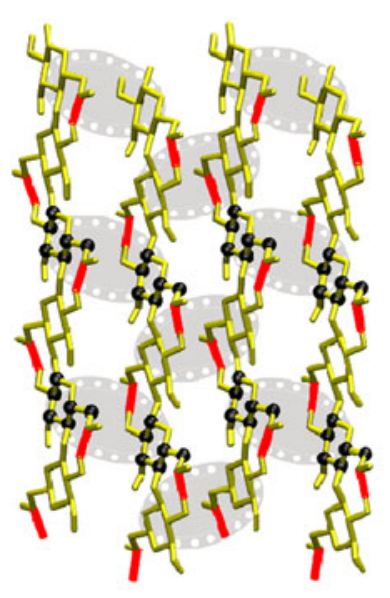

$\mathrm{I} \beta$

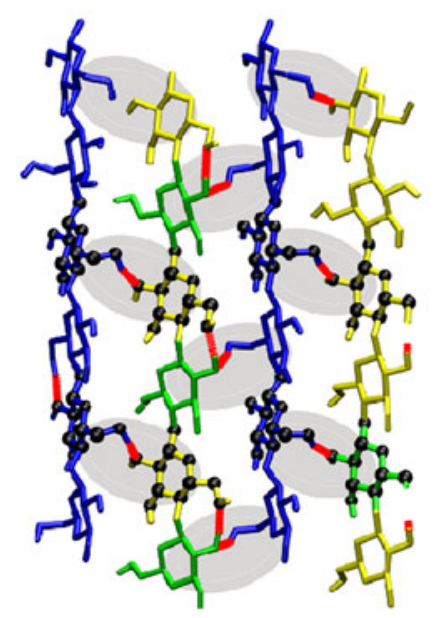

I-HT

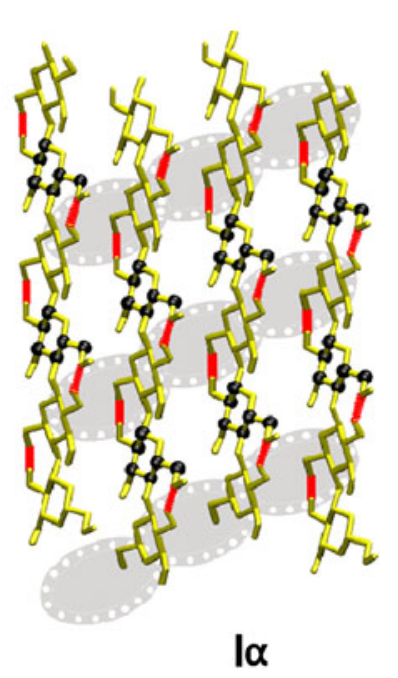

Fig. 5 Chains from four neighboring layers of cellulose $\mathrm{I} \beta$, I-HT and I $\alpha$. Two monomers in each chain are indicated with black spheres as in Fig. 4 . These chains belong to $(110)_{\beta, \mathrm{HT}}$ and $(100)_{\alpha}$ planes. Interlayer O6-O2 hydrogen bonds form at elevated temperatures. Monomer pairs close enough to form these O6-O2 interactions are circled in the bottom images. Hydrogen bonds involving both $\mathrm{O} 6$ and $\mathrm{O} 2$ are shown in red. In cellulose $\mathrm{I} \alpha$, it is not possible to form all of these possible interlayer interactions at the same time. See main text for

form where all possible $\mathrm{O} 6_{\text {center }}-\mathrm{O} 2_{\text {origin }}$ hydrogen bond pairs can form simultaneously. Upon cooling, kinetic trapping of $\mathrm{I} \beta$-like $\pm \mathbf{c} / 4$ layer packing can explain production of $\mathrm{I} \beta$ regardless of the relative free energy difference of I $\beta$ and $\mathrm{I} \alpha$. The symmetry and layer packing of the high temperature phase is I $\beta$-like, so we hypothesize the barrier to re-forming I $\alpha$-like layer packing is higher than keeping $\mathrm{I} \beta$-like layer packing. The reverse pathway of I $\beta$ to $\mathrm{I} \alpha$ via the high temperature intermediate is unlikely due to kinetic limitations.

\section{Discussion and conclusions}

Phase transformations via metastable intermediates may not directly reflect the relative thermodynamic description. At elevated temperatures, we hypothesize there is enough kinetic energy to overcome the barrier to chain slip in $\mathrm{I} \alpha$, which allows the cooperative alternating-by-layer chain tilt and $\mathrm{O} 6_{\text {center }}-\mathrm{O} 2_{\text {origin }}$ interlayer hydrogen bonding to form. Curved arrows indicate rotation sense of chain tilt as viewed along the chain axis with the non-reducing end near. Upon cooling, the barrier to forming $\mathrm{I} \beta$ is smaller than the barrier to chain slip and forming $\mathrm{I} \alpha$, so $\mathrm{I} \beta$ is preferentially formed due to kinetic rather than thermodynamic effects

stability of the end state polymorph forms due to kinetic limitations, where the timescale of the phase transformation rate to the more stable phase can be significantly longer than the accessible timescale of observation. The existence of metastable states is a well-known phenomenon in the crystallization and phase transitions of polymers (Cheng and Keller 1998; Rastogi and Kurelec 2000). We note that while it is tempting to conclude that the $\mathrm{I} \alpha$ phase is less thermodynamically stable than $\mathrm{I} \beta$ due to the production of $\mathrm{I} \beta$ upon cooling the high temperature phase, using this observation alone to assume a difference in free energy between cellulose $\mathrm{I} \alpha$ and $\mathrm{I} \beta$ is not necessarily correct.

Kinetics-not thermodynamics - may govern the selective crystallization of $\mathrm{I} \beta$ rather than $\mathrm{I} \alpha$ when 


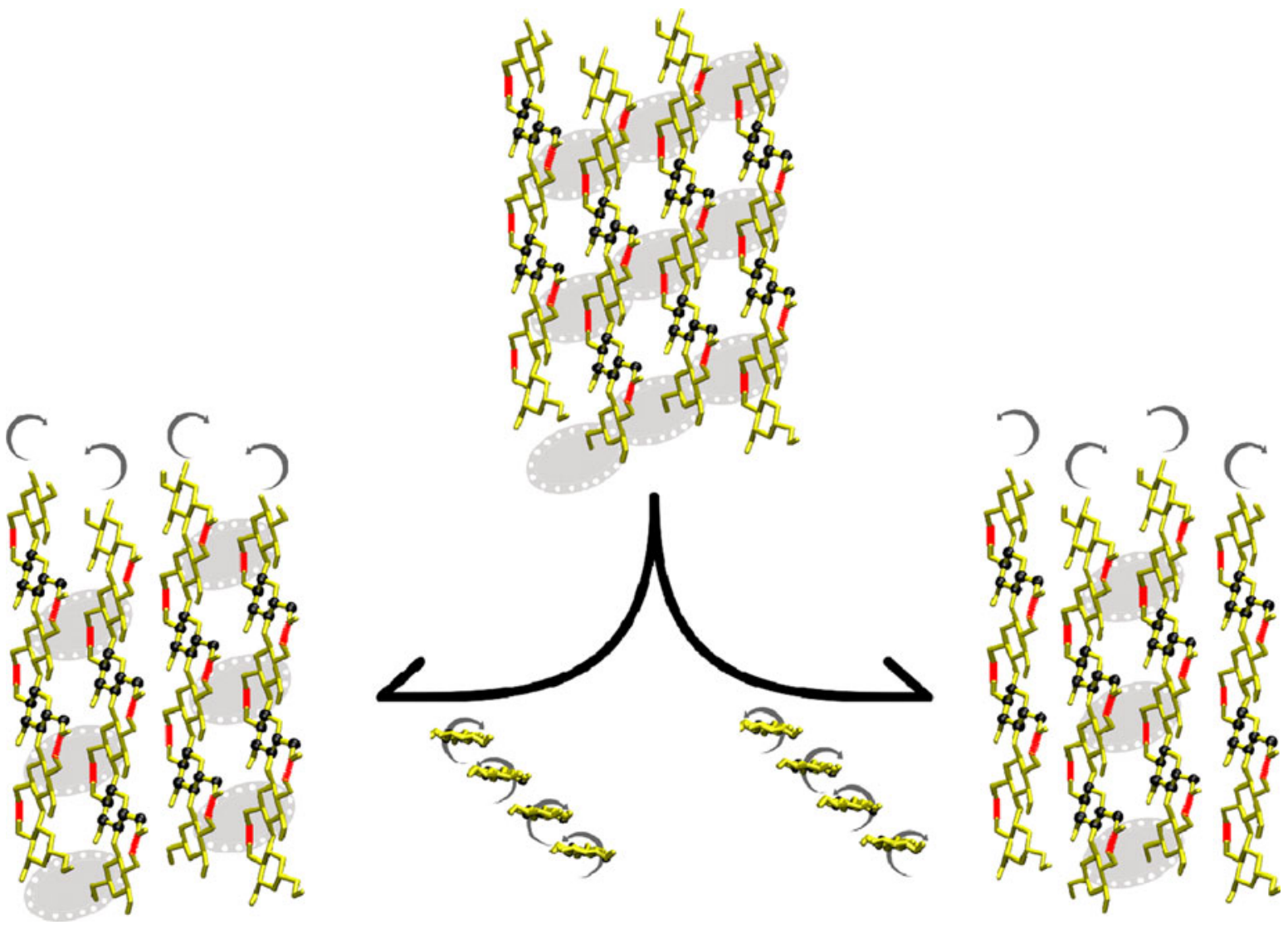

Fig. 6 For cellulose $\mathrm{I} \alpha$-like layer packing with chains tilting as in I-HT, each chain can potentially form O6-O2 hydrogen bond pairs with either the layer above or the layer below, but not with both at once. Cellulose $\mathrm{I} \alpha$-like layer packing is non-cooperative for the formation of O6-O2 interlayer hydrogen bonding at high temperature. If a monomer acts as the $\mathrm{O6}$ partner, the chain will

cooling from the high temperature phase. This is somewhat analogous to Ostwald's Rule of Stages where the least stable polymorph is crystallized first from nucleation to solution, and can be kinetically trapped at appropriate experimental conditions (Keller et al. 1994a; Threlfall 2003). Changing solvent or temperature can switch the order of relative polymorph stability, and are ways phase transformations can be achieved. Selective crystallization of cellulose polymorphs by varying temperature can be achieved by precipitation of a cellulose solution in $85 \%$ sulfuric acid into glycerol (Atalla and Whitmore 1978; Atalla et al. 1984). This procedure was reported to produce cellulose II at room temperature, cellulose IV at $150{ }^{\circ} \mathrm{C}$, and cellulose I at $170{ }^{\circ} \mathrm{C}$. Similarly dependent upon temperature, sharp cellulose I or IV diffraction peaks have been reported from low degree of tilt such that the same monomer cannot be the $\mathrm{O} 2$ partner for the next layer. This reduces the maximum number of O6-O2 interlayer hydrogen bonds able to form simultaneously. For a large crystal, at most half of the possible pairs can form at the same time, whereas I $\beta$-like layer packing allows all of these pairs to form cooperatively and simultaneously

polymerization (15-20 DP) crystals which were annealed over $2 \mathrm{~h}$ from precipitated low DP cellulose II, but it is not clear whether this material was cellulose $\mathrm{IV}_{\text {I }}$ or IV $\mathrm{II}_{\text {II }}$ (Atalla et al. 1984). The Raman spectrum of this low DP cellulose IV material appeared similar to parts of both cellulose I and cellulose II spectra.

Generalizing this proposed mechanism of solidsolid phase transformation pathways via metastable intermediate states provides insight to other cellulose polymorph transformations. The conversion of cellulose I to cellulose III $_{\text {I }}$ proceeds via metastable intermediates containing guest molecules such as ammonia or ethylenediamine (Wada et al. 2004a; Wada et al. 2006; Wada et al. 2009; Wada et al. 2010). The intermediate states have the GT conformation and layer packing similar to cellulose III $_{\mathrm{I}}$, so kinetic effects can favor formation of cellulose III $_{I}$ rather than 
cellulose I when the guest molecules are removed. The metastable intermediate structures can be viewed as primed to form cellulose III $_{\mathrm{I}}$. A more complicated phase diagram for the conversion of cellulose I to cellulose II via Mercerization exists, where there are several intermediate states depending upon concentration of $\mathrm{NaOH}$ and temperature (Roy et al. 2001; Dinand et al. 2002; Langan et al. 2005; Porro et al. 2007; Schoeck et al. 2007; Zugenmaier 2007; Kobayashia et al. 2011). The transformation of cellulose $\mathrm{III}_{\mathrm{I}}$ to $\mathrm{I} \beta$ or to $\mathrm{IV}_{\mathrm{I}}$ is also sensitive to temperature, and deserves further study (Wada 2001). One report indicates heating cellulose $\mathrm{III}_{\mathrm{I}}$ in glycerol to an intermediate temperature of $180{ }^{\circ} \mathrm{C}$ produces a combination of $\mathrm{I}$ and $\mathrm{IV}_{\mathrm{I}}$ diffraction peaks, whereas heating to a temperature of $210{ }^{\circ} \mathrm{C}$ results in sharp cellulose $\mathrm{IV}_{\mathrm{I}}$ diffraction peaks only (Sueoka et al. 1973). This is in contrast to more recent investigations, which suggest broad cellulose $\mathrm{IV}_{\mathrm{I}}$ diffraction peaks are artifacts due to lateral disorder or small crystallite diameter (Wada et al. 2004b; Newman 2008). The I-HT structure has some similarity to a structure with lattice parameters like those proposed for cellulose $\mathrm{IV}_{\mathrm{I}}$ (Gardiner and Sarko 1985; Matthews et al. 2006). The presence of three sharp diffraction peaks for materials produced under certain conditions indicates the existence of an orthorhombic lattice together with monoclinic or triclinic lattices (i.e., both cellulose $\mathrm{IV}_{\mathrm{I}}$ and cellulose I are present) (Sueoka et al. 1973). Kinetic trapping under certain conditions may account for observations of sharp cellulose $\mathrm{IV}_{\mathrm{I}}$ diffraction peaks, while small microfibril diameter may account for the broad "cellulose $I V_{I}$ " diffraction peaks observed for materials produced under different conditions (Sueoka et al. 1973; Chidambareswaran et al. 1982; Atalla et al. 1984; Chanzy et al. 1986; Helbert et al. 1997; Wada et al. 2004b).

Predicting the relative free energy of polymorphs with molecular simulation is challenging (Karamertzanis et al. 2008; Price 2008). Using single structures to calculate the zero point energy of bulk unit cells at $0 \mathrm{~K}$ may not give the best estimate of the relative free energy ranking of finite sized crystals at higher temperatures (van Eijck and Mooij 1995; Verwer and Leusen 2007; van Eijck 2001; van Eijck et al. 2001; Coombes et al. 2002; Woodley and Catlow 2008; Li et al. 2011). Entropic effects may play a role in the relative stability of polymorphs at a given temperature. Hydrogen bond and primary alcohol conformational disorder exist in cellulose $\mathrm{I} \alpha$ and $\mathrm{I} \beta$, and may affect the relative stability of these polymorphs (Langan et al. 2005; Yamamoto et al. 2006). With molecular simulation, achieving adequate sampling of conformational space while calculating accurate energies for each conformation can be conflicting goals. Molecular dynamics simulations of finite size cellulose crystals with pair-wise additive force fields can sample many conformations at the expense of electronic structure accuracy. More accurate quantum mechanical calculations are practical only for smaller numbers of atoms, and it is more difficult to account for the effects of conformational disorder. Crystal size and morphology can also affect the stability of polymorphs, so stability predictions from simulation should take this into account (Keller et al. 1994a; b).

The issue of polymorph thermodynamic stability can be resolved experimentally by calorimetric studies where the polymorphs are directly dissolved to a common solution state (Wadso and Goldberg 2001). This is challenging for cellulose polymorphs because of the need to compare polymer crystals with the same degree of polymerization, and which are arranged into microfibrils with the same number of chains, and which have the same cross-section morphology. A study has been conducted comparing the heats of crystallization of cellulose I and II, however, it is not clear whether the above criteria were met (Dale and Tsao 1982). Comparing cellulose $\mathrm{I} \alpha$ and I $\beta$ microfibrils of a given size and morphology with calorimetric measurements may confirm the widely held belief that $\mathrm{I} \beta$ is lower in free energy than $\mathrm{I} \alpha$, but this is still an open question.

Acknowledgments This material is based upon work supported as part of the Center for Direct Catalytic Conversion of Biomass to Biofuels (C3Bio), an Energy Frontier Research Center funded by the U.S. Department of Energy, Office of Science, Office of Basic Energy Sciences under Award Number DE-SC0000997. The authors would like to thank Gregg T. Beckham for helpful discussions.

\section{References}

Atalla RH, Whitmore RE (1978) Influence of elevated-temperatures on structure in isolation of native cellulose. J Polym Sci Part C Poly Lett 16(11):601-605

Atalla RH, Ellis JD, Schroeder LR (1984) Some effects of elevated-temperatures on the structure of cellulose and its transformation. J Wood Chem Technol 4(4):465-482 
Beckham G, Matthews JF, Peters B, Bomble YJ, Himmel M, Crowley M (2011) Molecular-level origins of biomass recalcitrance: decrystallization free energies for four common cellulose polymorphs. J Phys Chem B 115(14): 4118-4127

Boisset C, Chanzy H, Henrissat B, Lamed R, Shoham Y, Bayer E (1999) Digestion of crystalline cellulose substrates by the Clostridium thermocellum cellulosome: structural and morphological aspects. Biochem J 340(Pt 3):829

Chanzy H, Henrissat B, Vuong R, Revol JF (1986) Structuralchanges of cellulose crystals during the reversible transformation cellulose-I reversible cellulose-III in Valonia. Holzforschung 40:25-30

Cheng SZD, Keller A (1998) The role of metastable states in polymer phase transitions: concepts, principles, and experimental observations. Annu Rev Mater Sci 28: $533-562$

Chidambareswaran PK, Sreenivasan S, Patil NB, Lokhande HT (1982) Further-studies on cellulose-III polymorphstransformations to cellulose-IV lattices and subsequent reactions. J Appl Polym Sci 27(2):709-730

Coombes DS, Catlow CRA, Gale JD, Hardy MJ, Saunders MR (2002) Theoretical and experimental investigations on the morphology of pharmaceutical crystals. J Pharm Sci 91(7): $1652-1658$

Dale BE, Tsao GT (1982) Crystallinity and heats of crystallization of cellulose-a micro-calorimetric investigation. J Appl Polym Sci 27(4):1233-1241

Debzi EM, Chanzy H, Sugiyama J, Tekely P, Excoffier G (1991) The $\mathrm{I} \alpha \rightarrow \mathrm{I} \beta$ transformation of highly crystalline cellulose by annealing in various media. Macromolecules 24(26): 6816-6822

Dinand E, Vignon M, Chanzy H, Heux L (2002) Mercerization of primary wall cellulose and its implication for the conversion of cellulose I $\rightarrow$ cellulose II. Cellulose 9(1): 7-18

Gardiner ES, Sarko A (1985) Packing analysis of carbohydrates and polysaccharides. The Crystal Strucutres of Celluloses $\mathrm{IV}_{\mathrm{I}}$ and $\mathrm{IV}_{\mathrm{II}}$. Canadian journal of chemistry, XVI

Hardy BJ, Sarko A (1996) Molecular dynamics simulations and diffraction-based analysis of the native cellulose fibre: structural modelling of the I- $\alpha$ and I- $\beta$ phases and their interconversion. Polymer 37(10):1833-1839

Hayashi N, Sugiyama J, Okano T, Ishihara M (1997a) Selective degradation of the cellulose I- $\alpha$ component in Cladophora cellulose with Trichoderma viride cellulase. Carbohydr Res 305(1):109-116

Hayashi N, Sugiyama J, Okano T, Ishihara M (1997b) The enzymatic susceptibility of cellulose microfibrils of the algal-bacterial type and the cotton-ramie type. Carbohydr Res 305(2):261-269

Helbert W, Sugiyama J, Ishihara M, Yamanaka S (1997) Characterization of native crystalline cellulose in the cell walls of oomycota. J Biotechnol 57(1-3):29-37

Horii F, Yamamoto H, Kitamaru R, Tanahashi M, Higuchi T (1987) Transformation of native cellulose crystals induced by saturated steam at high-temperatures. Macromolecules 20(11):2946-2949

Horikawa Y, Sugiyama J (2009) Localization of crystalline allomorphs in cellulose microfibril. Biomacromolecules 10(8):2235-2239
Horikawa Y, Clair B, Sugiyama J (2009) Varietal difference in cellulose microfibril dimensions observed by infrared spectroscopy. Cellulose 16(1):1-8

Imai T, Sugiyama J (1998) Nanodomains of I- $\alpha$ and I- $\beta$ cellulose in algal microfibrils. Macromolecules 31(18):6275-6279

Karamertzanis PG, Raiteri P, Parrinello M, Leslie M, Price S (2008) The thermal stability of lattice-energy minima of 5-fluorouracil: metadynamics as an aid to polymorph prediction. J Phys Chem B 112(14):4298-4308

Keller A, Hikosaka M, Rastogi S, Toda A, Barham PJ, Goldbeck-Wood G (1994a) An approach to the formation and growth of new phases with application to polymer crystallization: effect of finite size, metastability, and Ostwald's rule of stages. J Mater Sci 29(10):2579-2604

Keller A, Hikosaka M, Rastogi S, Toda A, Barham PJ, Goldbeckwood G (1994b) The size factor in phase-transitionsits role in polymer crystal-formation and wider implications. Philos Trans Royal Soc Lond Ser A Math Phys Eng Sci 348(1686):3-17

Kobayashia K, Kimuraa S, Togawab E, Wada M (2011) Crystal transition from Na-cellulose IV to cellulose II monitored using synchrotron X-ray diffraction. Carbohydr Polym 83(2):483-488

Kono H, Numata Y (2006) Structural investigation of cellulose I- $\alpha$ and I- $\beta$ by 2D RFDR NMR spectroscopy: determination of sequence of magnetically inequivalent D-glucose units along cellulose chain. Cellulose 13(3):317-326

Langan P, Sukumar N, Nishiyama Y, Chanzy H (2005) Synchrotron X-ray structures of cellulose I- $\beta$ and regenerated cellulose II at ambient temperature and $100 \mathrm{~K}$. Cellulose 12(6):551-562

Li Y, Lin M, Davenport JW (2011) Ab initio studies of cellulose I: crystal structure, intermolecular forces, and interactions with water. J Phys Chem C 115(23):11533-11539

Matthews JF, Skopec C, Mason P, Zuccato P, Torget R, Sugiyama J, Himmel M, Brady J (2006) Computer simulation studies of microcrystalline cellulose I $\beta$. Carbohydr Res 341(1):138-152

Matthews JF, Bergenstrahle M, Beckham G, Himmel M, Nimlos MR, Brady J, Crowley M (2011) High-temperature behavior of cellulose I. J Phys Chem B 115(10):2155-2166

Newman R (2008) Simulation of X-ray diffractograms relevant to the purported polymorphs cellulose $\mathrm{IV}_{\mathrm{I}}$ and $\mathrm{IV}_{\mathrm{II}}$. Cellulose 15(6):769-778

Nishiyama Y, Langan P, Chanzy H (2002) Crystal structure and hydrogen-bonding system in cellulose $\mathrm{I} \beta$ from synchrotron $\mathrm{X}$-ray and neutron fiber diffraction. J Am Chem Soc 124(31):9074-9082

Nishiyama Y, Sugiyama J, Chanzy H, Langan P (2003) Crystal structure and hydrogen bonding system in cellulose $\mathrm{I} \alpha$, from synchrotron X-ray and neutron fiber diffraction. J Am Chem Soc 125(47):14300-14306

Porro Bedue, Chanzy H, Heux L (2007) Solid-state C-13 NMR study of Na-cellulose complexes. Biomacromolecules $8(8): 2586-2593$

Price S (2008) From crystal structure prediction to polymorph prediction: interpreting the crystal energy landscape. Phys Chem Chem Phys 10(15):1996-2009

Rastogi S, Kurelec L (2000) Polymorphism in polymers; its implications for polymer crystallisation. J Mater Sci 35(20):5121-5138 
Roy C, Budtova T, Navard P, Bedue O (2001) Structure of cellulose-soda solutions at low temperatures. Biomacromolecules 2(3):687-693

Sassi J, Tekely P, Chanzy H (2000) Relative susceptibility of the $\mathrm{I} \alpha$ and $\mathrm{I} \beta$ phases of cellulose towards acetylation. Cellulose 7(2):119-132

Schoeck J, Davies RJ, Martel A, Riekel C (2007) Na-cellulose formation in a single cotton fiber studied by synchrotron radiation microdiffraction. Biomacromolecules 8(2): 602-610

Sueoka A, Hayashi J, Watanabe S (1973) Mechanism of transformation into cellulose IV $\left(\mathrm{IV}_{\mathrm{I}}\right.$ and $\left.\mathrm{IV}_{\mathrm{II}}\right)$ from various crystalline modifications of cellulose. Nippon Kagaku Kaishi 7:1345-1352

Sugiyama J, Okano T, Yamamoto H, Horii F (1990) Transformation of Valonia cellulose crystals by an alkaline hydrothermal treatment. Macromolecules 23(12):3196-3198

Sugiyama J, Vuong R, Chanzy H (1991) Electron-diffraction study on the 2 crystalline phases occurring in native cellulose from an algal cell-wall. Macromolecules 24(14): 4168-4175

Threlfall T (2003) Structural and thermodynamic explanations of Ostwald's rule. Org Process Res Dev 7(6):1017-1027

van Eijck BP (2001) Ab initio crystal structure predictions for flexible hydrogen-bonded molecules. Part III. Effect of lattice vibrations. J Comput Chem 22(1):816-826

van Eijck BP, Mooij WTM (1995) Attempted prediction of the crystal structures of six monosaccharides. Acta Crystallogr Sect B 51(1):99-103

van Eijck BP, Mooij WTM, Kroon J (2001) Crystal structure prediction for six monosaccharides revisited. J Phys Chem B 105(43):10573-10578

Verwer P, Leusen FJJ (2007) Computer simulation to predict possible crystal polymorphs. In: Lipkowitz KB, Boyd DB (eds) Reviews in computational chemistry, vol 12. Wiley, USA

Vietor RJ, Mazeau K, Lakin M, Perez S (2000) A priori crystal structure prediction of native celluloses. Biopolymers 54(5): 342-354

Wada M (2001) In situ observation of the crystalline transformation from cellulose $\mathrm{III}_{\mathrm{I}}$ to $\mathrm{I} \beta$. Macromolecules 34(10): 3271-3275

Wada M, Okano T (2001) Localization of I- $\alpha$ and $\mathrm{I}-\beta$ phases in algal cellulose revealed by acid treatments. Cellulose 8(3): 183-188

Wada M, Kondo T, Okano T (2003) Thermally induced crystal transformation from cellulose I- $\alpha$ to I- $\beta$. Polym J 35(2): $155-159$
Wada M, Chanzy H, Nishiyama Y, Langan P (2004a) Cellulose $\mathrm{III}_{\mathrm{I}}$ crystal structure and hydrogen bonding by synchrotron $\mathrm{X}$-ray and neutron fiber diffraction. Macromolecules 37(23):8548-8555

Wada M, Heux L, Sugiyama J (2004b) Polymorphism of cellulose I family: reinvestigation of cellulose IV. Biomacromolecules 5(4):1385-1391

Wada M, Nishiyama Y, Langan P (2006) X-ray structure of ammonia-cellulose I: new insights into the conversion of cellulose I to cellulose III. Macromolecules 39(8): 2947-2952

Wada M, Heux L, Nishiyama Y, Langan P (2009) The structure of the complex of cellulose I with ethylenediamine by $\mathrm{X}$-ray crystallography and cross-polarization/magic angle spinning 13C nuclear magnetic resonance. Cellulose 16(6): 943-957

Wada M, Hori R, Kim U, Sasaki S (2010) X-ray diffraction study on the thermal expansion behavior of cellulose $\mathrm{I} \beta$ and its high-temperature phase. Polym Degrad Stab 95(8): 1330-1334

Wadso I, Goldberg RN (2001) Standards in isothermal microcalorimetry (IUPAC technical report). Pure Appl Chem 73(10): 1625-1639

Watanabe A, Morita S, Ozaki Y (2007) Temperature-dependent changes in hydrogen bonds in cellulose $\mathrm{I} \alpha$ studied by infrared spectroscopy in combination with perturbationcorrelation moving-window two-dimensional correlation spectroscopy: comparison with cellulose I $\beta$. Biomacromolecules 8(9):2969-2975

Woodley SM, Catlow CRA (2008) Crystal structure prediction from first principles. Nat Mater 7:937-946

Yamamoto H, Horii F, Odani H (1989) Structural-changes of native cellulose crystals induced by annealing in aqueous alkaline and acidic solutions at high-temperatures. Macromolecules 22(10):4130-4132

Yamamoto H, Horii F, Hirai A (2006) Structural studies of bacterial cellulose through the solid-phase nitration and acetylation by CP/MAS 13C NMR spectroscopy. Cellulose 13(3):327-342

Zhang Q, Bulone V, Ågren H, Tu Y (2011) A molecular dynamics study of the thermal response of crystalline cellulose I $\beta$. Cellulose 18(2):207-221

Zugenmaier P (2007) Crystalline cellulose and derivatives: characterization and structures. Springer, Berlin 